\title{
Global Stability of Two-Group Epidemic Models with Distributed Delays and Random Perturbation
}

\author{
Xiaoming Fan, Zhigang Wang, and Xuelian Xu \\ School of Mathematics Science, Harbin Normal University, Harbin 150025, China \\ Correspondence should be addressed to Zhigang Wang, wangzg2003205@yahoo.com.cn
}

Received 1 November 2011; Accepted 31 December 2011

Academic Editor: Norimichi Hirano

Copyright (C) 2012 Xiaoming Fan et al. This is an open access article distributed under the Creative Commons Attribution License, which permits unrestricted use, distribution, and reproduction in any medium, provided the original work is properly cited.

We discuss a two-group SEIR epidemic model with distributed delays, incorporating random fluctuation around the endemic equilibrium. Our research shows that the endemic equilibrium of the model with distributed delays and random perturbation is stochastically asymptotically stable in the large. In addition, a sufficient stability condition is obtained by constructing suitable Lyapunov function.

\section{Introduction}

For the research of control of disease in populations, significant progress has been made in the theory and application of epidemiology modeling by mathematical research [1-8]. One of the main problems for the theory of differential equations and their applications is connected with stability. Most traditional compartmental models in mathematical epidemiology descend from the classical SIR model of Kermack and McKendrick [9], where the population is divided into the classes of susceptible, infected, and recovered individuals. For some diseases, such as influenza and tuberculosis, on adequate contact with an infectious individual, a susceptible becomes exposed for a while, that is, infected but not yet infectious. Thus it is realistic to introduce a latent compartment; the total population can be partitioned into four compartments: susceptible, latent or exposed, infectious, and recovered, with sizes denoted by S, E, I, and R, respectively. The resulting models are of SEI, SEIR, or SEIRS type, respectively. SEIR model has been widely discussed in the literature. Local and global stability analysis of the disease-free and endemic equilibria has been carried out using different assumptions and contact rates in [6, 7]. Greenhalgh [5] considered SEIR models that incorporate density dependence in the death rate. Korobeinikov [6] considers the global properties for SEIR and SEIS by means of the Lyapunov functions. In fact, there are real 
benefits to be gained in using stochastic models because real life is full of randomness and stochasticity. Recently, some stochastic epidemic models have been studied by many authors, see $[3,10]$. Dalal et al. [4] showed that stochastic models had nonnegative solutions and carried out analysis on the asymptotic stability of models. Tornatore et al. [10] studied the stability of disease-free equilibrium of a stochastic SIR model with or without distributed time delay. On the other hand, taking into account environmental variability, white noise stochastic perturbations around the positive endemic equilibrium of epidemic models was considered in $[3,11]$. Beretta et al. proved the stability of epidemic model with stochastic time delays influenced by probability under certain conditions [3]. Such type of stochastic perturbations firstly was proposed in $[3,12]$ and later was successfully used in many other papers for many other different systems (see, for instance, [13-20]). A more general multigroup epidemic model is proposed to describe the disease spread in a heterogeneous host population with general age structure and varying infectivity by Li et al. [1]. They investigated a class of multigroup epidemic models with distributed delays and established the global dynamics determined by the basic reproduction number $R_{0}$. More specifically, they proved that, if $R_{0} \leq 1$, then the disease-free equilibrium is globally asymptotically stable; if $R_{0}>1$, then there exists a unique endemic equilibrium, and it is globally asymptotically stable. However, to the best of the authors' knowledge, no literature exists regarding SEIR model with random perturbation. Thus, the current study hopes to serve such a need and is inspired by the report of [1]. In this paper, based on the SEIR model of [1], we consider the white noise stochastic perturbations around its endemic equilibrium and use the methods, which is similar to [3]. We construct a class of the Lyapunov functions, as it is useful to study the global properties of stochastic models. By means of it, we prove the SEIR model is stochastically asymptotically stable in the large under certain condition.

The paper is organized as follows. In Section 2 we recall the deterministic SEIR model and its main results by $\mathrm{Li}$ et al. [1]. We introduce the model with stochastic perturbations around the endemic equilibrium in Section 3. In Section 4 the global stability of the endemic equilibrium is proved by the method of the Lyapunov functions.

\section{Preliminaries}

We briefly review the following results obtained by Li et al. [1]. Let $S_{k}, E_{k}, I_{k}$, and $R_{k}$ denote the susceptible, infected but noninfectious, infectious, and recovered populations in the $k$ th group, respectively. Let $i_{k}(t, r)$ denote the population of infectious individuals in the $k$ th group with respect to the age of infection $r$ at time $t$, and $I_{k}(t)=\int_{r=0}^{\infty} i_{k}(t, r) d r$. Let $h_{k}(r) \geqslant 0$ be a continuous kernel function that represents the infectivity at the age of infection $r$. The disease incidence in the $k$-th group, assuming a bilinear incidence form, can be calculated as $\sum_{j=1}^{n} \beta_{k j} S_{k}(t) \int_{r=0}^{\infty} h_{j}(r) i_{j}(t, r) d r$, where the sum takes into account cross-infections from all groups and $\beta_{k j}$ represents the transmission coefficient between compartments $S_{k}$ and $I_{j}$. In the special case $h_{k}(r) \equiv 1$, the incidence becomes $\sum_{j=1}^{n} \beta_{k j} S_{k}(t) I_{j}(t)$ as in [2]. Therefore, the model in [2] can be generalized to the following system of differential equations

$$
\begin{gathered}
S_{k}^{\prime}=\Lambda_{k}-\sum_{j=1}^{n} \beta_{k j} S_{k}(t) \int_{r=0}^{\infty} h_{j}(r) i_{j}(t, r) d r-d_{k}^{S} S_{k}, \\
E_{k}^{\prime}=\sum_{j=1}^{n} \beta_{k j} S_{k}(t) \int_{r=0}^{\infty} h_{j}(r) i_{j}(t, r) d r-\left(d_{k}^{E}+\epsilon_{k}\right) E_{k},
\end{gathered}
$$




$$
\begin{gathered}
I_{k}^{\prime}=\epsilon_{k} E_{k}-\left(d_{k}^{I}+\gamma_{k}\right) I_{k} \\
R_{k}^{\prime}=\gamma_{k} I_{k}-d_{k}^{R} R_{k}, \quad k=1,2, \ldots, n .
\end{gathered}
$$

Here $\Lambda_{k}$ represents influx of individuals into the $k$ th group, $d_{k}^{S}, d_{k}^{E}, d_{k}^{I}$, and $d_{k}^{R}$ represent death rates of $S, E, I$, and $R$ populations in the $k$ th group, respectively, $\epsilon_{k}$ represents the rate of becoming infectious after a latent period in the $k$-th group, and $\gamma_{k}$ represents the recovery rate of infectious individuals in the $k$-th group. All parameter values are assumed to be nonnegative and $\Lambda_{k}, d_{k}^{S}, d_{k}^{E}>0$ for all $k$. Note that

$$
\begin{aligned}
\left(\frac{\partial}{\partial t}+\frac{\partial}{\partial r}\right) i_{k}(t, r) & =-\left(d_{k}^{I}+\gamma_{k}\right) i_{k}(t, r), \\
i_{k}(t, 0) & =\epsilon_{k} E_{k}(t),
\end{aligned}
$$

whose solution is

$$
i_{k}(t, r)=i_{k}(t-r, 0) e^{-\left(d_{k}^{I}+\gamma_{k}\right) r}=\epsilon_{k} E_{k}(t-r) e^{-\left(d_{k}^{I}+\gamma_{k}\right) r} .
$$

Substituting (2.3) into (2), we obtain

$$
\begin{gathered}
S_{k}^{\prime}=\Lambda_{k}-\sum_{j=1}^{n} \beta_{k j} S_{k}(t) \int_{r=0}^{\infty} h_{j}(r) \epsilon_{j} E_{j}(t-r) e^{-\left(d_{j}^{I}+\gamma_{j}\right) r} d r-d_{k}^{S} S_{k}, \\
E_{k}^{\prime}=\sum_{j=1}^{n} \beta_{k j} S_{k}(t) \int_{r=0}^{\infty} h_{j}(r) \epsilon_{j} E_{j}(t-r) e^{-\left(d_{j}^{I}+\gamma_{j}\right) r} d r-\left(d_{k}^{E}+\epsilon_{k}\right) E_{k} \\
I_{k}^{\prime}=\epsilon_{k} E_{k}-\left(d_{k}^{I}+\gamma_{k}\right) I_{k}, \\
R_{k}^{\prime}=\gamma_{k} I_{k}-d_{k}^{R} R_{k}, \quad k=1,2, \ldots, n .
\end{gathered}
$$

Since the variables $I_{k}$ and $R_{k}$ do not appear in the first two equations of (2.4), Li et al. consider the following reduced system with distributed time delays and general kernel functions [1]:

$$
\begin{gathered}
S_{k}^{\prime}=\Lambda_{k}-\sum_{j=1}^{n} \beta_{k j} S_{k}(t) \int_{r=0}^{\infty} f_{j}(r) E_{j}(t-r) d r-d_{k}^{S} S_{k}, \\
E_{k}^{\prime}=\sum_{j=1}^{n} \beta_{k j} S_{k}(t) \int_{r=0}^{\infty} f_{j}(r) E_{j}(t-r) d r-\left(d_{k}^{E}+\epsilon_{k}\right) E_{k} .
\end{gathered}
$$

Here the kernel function $f_{k}(r) \geqslant 0$ is continuous and $1 \geqslant \int_{r=0}^{\infty} f_{\mathrm{k}}(r) d r=h_{k}>0$. System (2.5) can be interpreted as a multigroup model for an infectious disease whose latent period 
$r$ in hosts has a general probability density function $\left(1 / h_{k}\right) f_{k}(r) d r$, for the $k$-th group. Let $S_{k}^{0}=\Lambda_{k} / d_{k}^{S}, h_{k}=\int_{r=0}^{\infty} f_{k}(r) d r$. The next-generation matrix for system (2.5) is

$$
M_{0}=\left(\frac{\beta_{k j} S_{k}^{0} h_{k}}{d_{k}^{E}+\epsilon_{k}}\right)_{n \times n}
$$

Define the basic reproduction number as the spectral radius of $M_{0}$,

$$
R_{0}=\rho\left(M_{0}\right)
$$

In the special case when $f_{k}(r)$ is an exponential function, $R_{0}$ reduces to that for the resulting ODE models. Make the following assumption on the kernel function $f_{k}(r)$ in (2.5):

$$
\int_{r=0}^{\infty} f_{k}(r) e^{-\lambda_{k} r} d r<\infty
$$

where $\lambda_{k}$ is a positive number, $k=1,2, \ldots, n$. Define the following Banach space of fading memory type:

$$
C_{k}=\left\{\phi \in((-\infty, 0], \mathbb{R}) \phi(s) e^{\lambda_{k} s} \text { is uniformly continuous on }(-\infty, 0] \text { and } \sup _{s \leqslant 0}|\phi(s)| e^{\lambda_{k} s}<\infty\right\}
$$

with norm $\|\phi\|_{k}=\sup _{s \leqslant 0}|\phi(s)| e^{\lambda_{k} s}$. For $\phi \in C_{k}$, let $\phi_{t} \in C_{k}$ be such that $\phi_{t}(s)=\phi(t+s), s \in$ $(-\infty, 0]$. Let $S_{k, 0} \in \mathbb{R}_{+}$and $\phi_{k} \in C_{k}$ such that $\phi_{k}(s) \geqslant 0, s \in(-\infty, 0]$. We consider solutions $\left(S_{1}(t), E_{1 t}, S_{2}(t), E_{2 t}, \ldots, S_{n}(t), E_{n t}\right)$ of system (2.5) with initial conditions

$$
S_{k}(0)=S_{k, 0}, \quad E_{k, 0}=\phi_{k}, \quad k=1,2, \ldots, n
$$

Standard theory of functional differential equations implies $E_{k t} \in C_{k}$ for $t>0$. We consider system (2.5) in the phase space

$$
X=\prod_{k=1}^{n}\left(\mathbb{R} \times C_{k}\right)
$$

It can be verified that solutions of (2.5) in $X$ initial conditions (2.10) remain nonnegative. In particular, $S_{k}(t)>0$ for $\mathrm{t}>0$. The following set is positively invariant for system (2.5):

$$
\begin{gathered}
\Theta=\left\{\left(S_{1}, E_{2}(\cdot), S_{2}, E_{2}(\cdot), \ldots, S_{n}, E_{n}(\cdot)\right) \in X \mid 0 \leqslant S_{k} \leqslant \frac{\Lambda_{k}}{d_{k}^{S}}, 0 \leqslant S_{k}+E_{k}(0) \leqslant \frac{\Lambda_{k}}{d_{k}^{*}}, E_{k}(s) \geqslant 0,\right. \\
s \in(-\infty, 0], k=1,2, \ldots, n\} .
\end{gathered}
$$


All positive semiorbits in $\Theta$ are precompact in $X$ and thus have nonempty $\omega$-limit sets. We have the following results [1].

Lemma 2.1. All positive semi-orbits in $\Theta$ have non-empty $\omega$-limit sets. Let

$$
\begin{aligned}
& \Theta^{\circ}=\left\{\left(S_{1}, E_{2}(\cdot), S_{2}, E_{2}(\cdot), \ldots, S_{n}, E_{n}(\cdot)\right) \in X \mid 0<S_{k}<\frac{\Lambda_{k}}{d_{k}^{S}}, 0<S_{k}+E_{k}(0)<\frac{\Lambda_{k}}{d_{k}^{*}}, E_{k}(s)>0,\right. \\
& \quad s \in(-\infty, 0], k=1,2, \ldots, n\} .
\end{aligned}
$$

It can be shown that $\Theta^{\circ}$ is the interior of $\Theta$.

Lemma 2.2. Assume that $B=\left(\beta_{k j}\right)$ is irreducible.

(1) If $R_{0} \leqslant 1$, then $P_{0}$ is the only equilibrium for system (2.5) in $\Theta$.

(2) If $R_{0}>1$, then there exist two equilibria for system (2.5) in $\Theta$ : the disease-free equilibrium $P_{0}$ and a unique endemic equilibrium $P^{*}$.

Lemma 2.3. Assume that $B=\left(\beta_{k j}\right)$ is irreducible.

(1) If $R_{0} \leqslant 1$, then the disease-free equilibrium $P_{0}$ of system (2.5) is globally asymptotically stable in $\Theta$. If $R_{0}>1$, then $P_{0}$ is unstable.

(2) If $R_{0}>1$, then the endemic equilibrium $P^{*}$ of system (2.5) is globally asymptotically stable in $\Theta^{\circ}$.

Biologically, Lemma 2.3 implies that if the basic reproduction number $R_{0} \leqslant 1$, then the disease always dies out from all groups; if $R_{0}>1$, then the disease always persists in all groups at the unique endemic equilibrium level, irrespective of the initial conditions.

\section{Stochastic Model Derivation}

In this paper, based on system (2.5), we consider the case of $k=1,2$ in the following system(3.1):

$$
\begin{gathered}
\dot{S}_{k}=\Lambda_{k}-\sum_{j=1}^{2} \beta_{k j} S_{\mathrm{k}} \int_{r=0}^{\infty} f_{j}(r) E_{j}(t-r) d r-d_{k}^{S} S_{k}, \\
\dot{E}_{k}=\sum_{j=1}^{2} \beta_{k j} S_{k} \int_{r=0}^{\infty} f_{j}(r) E_{j}(t-r) d r-\left(d_{k}^{E}+\epsilon_{k}\right) E_{k}, \\
\dot{I}_{k}=\epsilon_{k} E_{k}-\left(d_{k}^{I}+\gamma_{k}\right) I_{k}, \\
\dot{R}_{k}=\gamma_{k} I_{k}-d_{k}^{R} R_{k} .
\end{gathered}
$$


It is easy to see that equilibrium for system (3.1) is given by $P^{*}=\left(S^{*} ; E^{*} ; I^{*} ; R^{*}\right)$,

$$
S_{k}^{*}=\frac{d_{k}^{E}+\epsilon_{k}}{\sum_{j=1}^{2} \beta_{k j} h_{j}}, \quad E_{k}^{*}=\frac{\Lambda_{k}-d_{k}^{S}}{\sum_{j=1}^{2} \beta_{k j} S_{k}^{*} h_{j}}, \quad I_{k}^{*}=\frac{\epsilon_{k} E_{k}^{*}}{d_{k}^{I}+\gamma_{k}}, \quad R_{k}^{*}=\frac{\gamma_{k} I_{k}^{*}}{d_{k}^{R}}
$$

We assume stochastic perturbations are of white noise type, which are directly proportional to distances $S_{k}(t), E_{k}(t), I_{k}(t), R_{k}(t)$ from values of $S_{k^{\prime}}^{*} E_{k^{\prime}}^{*} I_{k^{\prime}}^{*} R_{k^{\prime}}^{*}$ influence the $\dot{S}_{k}(t), \dot{E}_{k}(t), \dot{I}_{k}(t), \dot{R}_{k}(t)$ respectively. So system (2.4) results in

$$
\begin{gathered}
\dot{S}_{k}=\Lambda_{k}-\sum_{j=1}^{2} \beta_{k j} S_{k} \int_{r=0}^{\infty} f_{j}(r) E_{j}(t-r) d r-d_{k}^{S} S_{k}+\sigma_{1 k}\left(S_{k}-S_{k}^{*}\right) \dot{B}_{1 k} \\
\dot{E}_{k}=\sum_{j=1}^{2} \beta_{k j} S_{k} \int_{r=0}^{\infty} f_{j}(r) j E_{j}(t-r) d r-\left(d_{k}^{E}+\epsilon_{k}\right) E_{k}+\sigma_{2 k}\left(E_{k}-E_{k}^{*}\right) \dot{B}_{2 k}, \\
\dot{I}_{k}=\epsilon_{k} E_{k}-\left(d_{k}^{I}+\gamma_{k}\right) I_{k}+\sigma_{3 k}\left(I_{k}-I_{k}^{*}\right) \dot{B}_{3 k}, \\
\dot{R}_{k}=\gamma_{k} I_{k}-d_{k}^{R} R_{k}+\sigma_{4 k}\left(R_{k}-R_{k}^{*}\right) \dot{B}_{4 k}, \quad k=1,2,
\end{gathered}
$$

where $B_{1 k}(t), B_{2 k}(t), B_{3 k}(t), B_{4 k}(t)$ are independent standard Brownian motions and $\sigma_{i k}^{2}>0$ represent the intensities of $B_{i k}(t)(i=1,2,3,4)$, respectively. Obviously, stochastic system (3.3) has the same equilibrium points as system (3.1). In the next section, we will investigate the stability of the equilibrium $P^{*}$ of system (3.3). Below we will construct a class of different Lyapunov functions to achieve our proof under certain conditions.

\section{Stochastic Stability of the Endemic Equilibrium}

In this paper, unless otherwise specified, let $\left(\Omega, \mathcal{F},\left\{\mathcal{F}_{t}\right\}_{t \geqslant t_{0}}, P\right)$ be a complete probability space with a filtration $\left\{\mathcal{F}_{t}\right\}_{t \geqslant t_{0}}$ satisfying the usual conditions (i.e., it is increasing and right continuous while $\mathcal{F}_{0}$ contains all $P$-null sets). Let $\beta_{i}(t)$ be the Brownian motions defined on this probability space. If $R_{0}>1$, then the stochastic system (3.3) can be centered at its endemic equilibrium $P^{*}=\left(S_{k}^{*}, E_{k}^{*}, I_{k}^{*}, R_{k}^{*}\right)$, by the change of variables

$$
u_{k}=S_{k}-S_{k^{\prime}}^{*} \quad v_{k}=E_{k}-E_{k^{\prime}}^{*} \quad w_{k}=I_{k}-I_{k^{\prime}}^{*} \quad z_{k}=R_{k}-R_{k}^{*}
$$

we obtain

$$
\begin{aligned}
\dot{u}_{k}= & -\sum_{j=1}^{2} \beta_{k j} u_{k} \int_{r=0}^{\infty} f_{j}(r) v_{j}(t-r) d r-\sum_{j=1}^{2} \beta_{k j} S_{k}^{*} \int_{r=0}^{\infty} f_{j}(r) v_{j}(t-r) d r-\sum_{j=1}^{2} \beta_{k j} u_{k} E_{j}^{*} h_{j}-d_{k}^{S} u_{k} \\
& +\sigma_{1 k} u_{k} \dot{B}_{1 k}, \\
\dot{v}_{k}= & \sum_{j=1}^{2} \beta_{k j} u_{k} \int_{r=0}^{\infty} f_{j}(r) v_{j}(t-r) d r+\sum_{j=1}^{2} \beta_{k j} S_{k}^{*} \int_{r=0}^{\infty} f_{j}(r) v_{j}(t-r) d r+\sum_{j=1}^{2} \beta_{k j} u_{k} E_{j}^{*} h_{j} \\
& -\left(d_{k}^{E}+\epsilon_{k}\right) v_{k}+\sigma_{2 k} v_{k} \dot{B}_{2 k},
\end{aligned}
$$




$$
\begin{aligned}
\dot{w}_{k} & =\epsilon_{k} v_{k}-\left(d_{k}^{I}+\gamma_{k}\right) w_{k}+\sigma_{3 k} w_{k} \dot{B}_{3 k}, \\
\dot{z}_{k} & =\gamma_{k} w_{k}-d_{k}^{R} z_{k}+\sigma_{4 k} z_{k} \dot{B}_{4 k}, \quad k=1,2 .
\end{aligned}
$$

It is easy to see that the stability of the equilibrium of the system (3.3) is equivalent to the stability of zero solution of system (4.2). Before proving the main theorem we put forward a lemma in [21]. Consider the $d$-dimensional stochastic differential equation

$$
d \mathbf{x}(t)=f(\mathbf{x}(t), t) d t+g(\mathbf{x}(t), t) d B(t), \quad t \geqslant t_{0} .
$$

Assume that the assumptions of the existence-and-uniqueness theorem are fulfilled. Hence, for any given initial value $\mathbf{x}\left(t_{0}\right)=\mathbf{x}_{0} \in R^{d},(4.3)$ has a unique global solution that is denoted by $\mathbf{x}\left(t ; t_{0}, x_{0}\right)$. Assume furthermore that $f(0, t)=0$ and $g(0, t)=0$ for all $t \geqslant t_{0}$. So (4.3) has the solution $\mathbf{x}(t) \equiv 0$ corresponding to the initial value $\mathbf{x}\left(t_{0}\right)=0$. This solution is called the trivial solution or equilibrium position. Denote by $C^{2,1}\left(R^{d} \times\left[t_{0}, \infty\right) ; R_{+}\right)$the family of all nonnegative functions $V(\mathbf{x}, t)$ defined on $R^{d} \times\left[t_{0}, \infty\right)$ such that they are continuously twice differentiable in $\mathbf{x}$ and once in $t$. Define the differential operator $L$ associated with (4.3) by

$$
L=\frac{\partial}{\partial t}+\sum_{i=1}^{d} f_{i}(\mathbf{x}, t) \frac{\partial}{\partial x_{i}}+\frac{1}{2} \sum_{i, j=1}^{d}\left[g^{T}(\mathbf{x}, t) g(\mathbf{x}, t)\right]_{i j} \frac{\partial^{2}}{\partial x_{i} x_{j}}
$$

If $L$ acts on a function $V \in C^{2,1}\left(R^{d} \times\left[t_{0}, \infty\right) ; R_{+}\right)$, then

$$
L V(\mathbf{x}, t)=V_{t}(\mathbf{x}, t)+V_{t}(\mathbf{x}, t) f(\mathbf{x}, t)+\frac{1}{2} \operatorname{Tr}\left[g^{T}(\mathbf{x}, t) V_{x x}(\mathbf{x}, t) g(\mathbf{x}, t)\right] .
$$

Definition 4.1. (1) The trivial solution of (4.3) is said to be stochastically stable or stable in probability if for every pair of $\varepsilon \in(0,1)$ and $r>0$, there exists a $\delta=\delta\left(\varepsilon, r, t_{0}\right)>0$ such that

$$
P\left\{\left|\mathbf{x}\left(t ; t_{0}, x_{0}\right)\right|<r \forall t \geqslant t_{0}\right\} \geqslant 1-\varepsilon
$$

whenever $\left|\mathbf{x}_{0}\right|<\delta$. Otherwise, it is said to be stochastically unstable.

(2) The trivial solution is said to be stochastically asymptotically stable if it is stochastically stable, and, moreover, for every $\varepsilon \in(0,1)$, there exists a $\delta_{0}=\delta_{0}\left(\varepsilon, t_{0}\right)>0$ such that

$$
P\left\{\lim _{t \rightarrow \infty} \mathbf{x}\left(t ; t_{0}, x_{0}\right)=0\right\} \geqslant 1-\varepsilon
$$

whenever $\left|\mathbf{x}_{0}\right|<\delta_{0}$.

(3)The trivial solution is said to be stochastically asymptotically stable in the large if it is stochastically asymptotically stable and, moreover, for all $\mathbf{x}_{0} \in R^{d}$,

$$
P\left\{\lim _{t \rightarrow \infty} \mathbf{x}\left(t ; t_{0}, x_{0}\right)=0\right\}=1
$$


Lemma 4.2 (see [21]). If there exists a positive-definite decrescent radially unbounded function $V(\mathbf{x}, t) \in C^{2,1}\left(R^{d} \times\left[t_{0}, \infty\right) ; R_{+}\right)$such that $L V(\mathbf{x}, t)$ is negative definite, then the trivial solution of (4.3) is stochastically asymptotically stable in the large.

From the above lemma, we can obtain the stochastically asymptotically stability of equilibrium as follows.

Theorem 4.3. Assume that $B=\left(\beta_{k j}\right)$ is irreducible and $R_{0}>1$; then, if the following condition is satisfied

$$
\sigma_{1 k}^{2}<2 d_{k^{\prime}}^{S} \quad \sigma_{2 k}^{2}<2\left(d_{k}^{E}+\epsilon_{k}\right), \quad \sigma_{3 k}^{2}<2\left(d_{k}^{I}+\gamma_{k}\right), \quad \sigma_{4 k}^{2}<2 d_{k^{\prime}}^{R}
$$

the endemic equilibrium $P^{*}$ of system (3.3) is stochastically asymptotically stable in the large.

Proof. It is easy to see that we only need to prove the zero solution of (4.2) is stochastically asymptotically stable in the large. Let $\mathbf{x}(t)=(u(t), v(t), w(t), z(t))^{T}$. We define the Lyapunov function $V(\mathbf{x}(t))$ as follows:

$$
V(\mathbf{x})=\sum_{k=1}^{2}\left[a_{k} u_{k}^{2}+v_{k}^{2}+b_{k}\left(u_{k}+v_{k}\right)^{2}+a_{k}^{2} w_{k}^{2}+a_{k}^{4} z_{k}^{2}+\sum_{j=1}^{2} \beta_{k j} S_{k}^{*}\left(1+a_{k}\right) \int_{r=0}^{\infty} f_{j}(r) \int_{t-r}^{t} v_{k}^{2}(\tau) d \tau d r\right],
$$

where $a_{k}>0, b_{k}>0$ are real positive constants to be chosen later. So it is obvious that $V(\mathbf{x})$ is positive definite and decrescent.

Using Itô's formula, we compute

$$
\begin{aligned}
& L V \\
& \qquad \sum_{k=1}^{2}\left\{2\left(a_{k} u_{k}+b_{k}\left(u_{k}+v_{k}\right)\right)\right. \\
& \times\left[-\sum_{j=1}^{2} \beta_{k j} u_{k} \int_{r=0}^{\infty} f_{j}(r) v_{j}(t-r) d r-\sum_{j=1}^{2} \beta_{k j} S_{k}^{*} \int_{r=0}^{\infty} f_{j}(r) v_{j}(t-r) d r-\sum_{j=1}^{2} \beta_{k j} u_{k} E_{j}^{*} h_{j}-d_{k}^{S} u_{k}\right] \\
& +2\left(v_{k}+b_{k}\left(u_{k}+v_{k}\right)\right)\left[\sum_{j=1}^{2} \beta_{k j} u_{k} \int_{r=0}^{\infty} f_{j}(r) v_{j}(t-r) d r+\sum_{j=1}^{2} \beta_{k j} S_{k}^{*} \int_{r=0}^{\infty} f_{j}(r) v_{j}(t-r) d r\right. \\
& \left.+2 \sum_{j=1}^{2} \beta_{k j} u_{k} E_{j}^{*} h_{j}-\left(d_{k}^{E}+\epsilon_{k}\right) v_{k}\right] \\
& \left.+a_{k}^{2} \sigma_{3 k}^{2} w_{k}^{2}+a_{k}^{4} \sigma_{4 k}^{2} z_{k}^{2}+\sum_{j=1}^{2} \beta_{k j} S_{k}^{*}\left(1+a_{k}\right) h_{j} v_{j}^{2}-\sum_{j=1}^{2} \beta_{k j} S_{k}^{*}\left(1+a_{k}\right) \int_{r=0}^{\infty} f_{j}(r) v_{j}^{2}(t-r) d r\right\} .
\end{aligned}
$$


Using (3.2), we obtain

$$
\begin{aligned}
L V=\sum_{k=1}^{2}\{- & u_{k}^{2}\left[\left(a_{k}+b_{k}\right)\left(2 d_{k}^{S}-\sigma_{1 k}^{2}\right)+2 \sum_{j=1}^{2} a_{k} \beta_{k j} E_{j}^{*} h_{j}\right]-v_{k}^{2}\left[\left(1+b_{k}\right)\left(2 \sum_{j=1}^{2} \beta_{k j} S_{k}^{*} h_{j}-\sigma_{2 k}^{2}\right)\right] \\
& -a_{k}^{2} w_{k}^{2}\left(2\left(d_{k}^{I}+\gamma_{k}\right)-\sigma_{3 k}^{2}\right)-a_{k}^{4} z_{k}^{2}\left(2 d_{k}^{R}-\sigma_{4 k}^{2}\right)+2\left(\sum_{j=1}^{2} \beta_{k j} E_{j}^{*} h_{j}-b_{k}\left(d_{k}^{S}+\sum_{j=1}^{2} \beta_{k j} S_{k}^{*} h_{j}\right)\right) \\
& \times u_{k} v_{k}+2\left(a_{k}^{2} \epsilon_{k} v_{k} w_{k}+\gamma_{k} w_{k} z_{k}\right)+2 \sum_{j=1}^{2} \beta_{k j} S_{k}^{*}\left(v_{k}-a_{k} u_{k}\right) \int_{r=0}^{\infty} f_{j}(r) v_{j}(t-r) d r \\
& +2 \sum_{j=1}^{2} \beta_{k j} u_{k}\left(v_{k}-a_{k} u_{k}\right) \int_{r=0}^{\infty} f_{j}(r) v_{j}(t-r) d r+\sum_{j=1}^{2} \beta_{k j} S_{k}^{*}\left(1+a_{\mathrm{k}}\right) h_{j} v_{j}^{2} \\
& \left.-\sum_{j=1}^{2} \beta_{k j} S_{k}^{*}\left(1+a_{k}\right) \int_{r=0}^{\infty} f_{j}(r) v_{j}^{2}(t-r) d r\right\} .
\end{aligned}
$$

In (4.12), we choose

$$
b_{k}=\frac{\sum_{j=1}^{2} \beta_{k j} S_{k}^{*} h_{j}}{\left(d_{k}^{S}+\sum_{j=1}^{2} \beta_{k j} S_{k}^{*} h_{j}\right)} .
$$

Then

$$
\begin{aligned}
L V=\sum_{k=1}^{2}\{- & u_{k}^{2}\left[\left(a_{k}+b_{k}\right)\left(2 d_{k}^{S}-\sigma_{1 k}^{2}\right)+2 \sum_{j=1}^{2} a_{k} \beta_{k j} E_{j}^{*} h_{j}\right]-v_{k}^{2}\left[\left(1+b_{k}\right)\left(2 \sum_{j=1}^{2} \beta_{k j} S_{k}^{*} h_{j}-\sigma_{2 k}^{2}\right)\right] \\
& -a_{k}^{2} w_{k}^{2}\left(2\left(d_{k}^{I}+\gamma_{k}\right)-\sigma_{3 k}^{2}\right)-a_{k}^{4} z_{k}^{2}\left(2 d_{k}^{R}-\sigma_{4 k}^{2}\right)+2 a_{k}^{2}\left(\epsilon_{k} v_{k} w_{k}+a_{k}^{2} \gamma_{k} w_{k} z_{k}\right) \\
& +2 \sum_{j=1}^{2} \beta_{k j} S_{k}^{*}\left(v_{k}-a_{k} u_{k}\right) \int_{r=0}^{\infty} f_{j}(r) v_{j}(t-r) d r+2 \sum_{j=1}^{2} \beta_{k j} u_{k}\left(v_{k}-a_{k} u_{k}\right) \int_{r=0}^{\infty} f_{j}(r) v_{j}(t-r) d r \\
& \left.+\sum_{j=1}^{2} \beta_{k j} S_{k}^{*}\left(1+a_{k}\right) h_{j} v_{j}^{2}-\sum_{j=1}^{2} \beta_{k j} S_{k}^{*}\left(1+a_{k}\right) \int_{r=0}^{\infty} f_{j}(r) v_{j}^{2}(t-r) d r\right\} .
\end{aligned}
$$

Moreover, using the Cauchy inequality to $2 a_{k}^{2} \epsilon_{k} v_{k} w_{k}$ and $2 a_{k}^{4} \gamma_{k} w_{k} z_{k}$, we can obtain

$$
\begin{aligned}
& 2 a_{k}^{2} \epsilon_{k} v_{k} w_{k} \leqslant a_{k}^{2} \epsilon_{k}\left(\frac{v_{k}^{2}}{a_{k}}+a_{k} w_{k}^{2}\right), \\
& 2 a_{k}^{4} \gamma_{k} w_{k} z_{k} \leqslant a_{k}^{4} \gamma_{k}\left(\frac{w_{k}^{2}}{a_{k}}+a_{k} z_{k}^{2}\right),
\end{aligned}
$$




$$
\begin{aligned}
2 \sum_{j=1}^{2} \beta_{k j} S_{k}^{*}\left(v_{k}-a_{k} u_{k}\right) \int_{r=0}^{\infty} f_{j}(r) v_{j}(t-r) d r \leqslant & a_{k} \sum_{j=1}^{2} \beta_{k j} S_{k}^{*}\left(u_{k}^{2}+\int_{r=0}^{\infty} f_{j}(r) v_{j}^{2}(t-r) d r\right) \\
& +\sum_{j=1}^{2} \beta_{k j} S_{k}^{*}\left(v_{k}^{2}+\int_{r=0}^{\infty} f_{j}(r) v_{j}^{2}(t-r) d r\right) .
\end{aligned}
$$

Substituting (4.15) into (4.14) as well as using $1 \geqslant \int_{r=0}^{\infty} f_{k}(r) d r=h_{k}>0$, yields

$$
\begin{aligned}
& L V \leqslant \sum_{k=1}^{2}\left\{-u_{k}^{2}\right. {\left[b_{k}\left(2 d_{k}^{S}-\sigma_{1 k}^{2}\right)-\sum_{j=1}^{2} a_{k} \beta_{k j} S_{k}^{*}\right] } \\
&-v_{k}^{2}\left[\left(1+b_{k}\right)\left(2 \sum_{j=1}^{2} \beta_{k j} S_{k}^{*}-\sigma_{2 k}^{2}\right)-a_{k}\left(\epsilon_{k}+\sum_{j=1}^{2} \beta_{k j} S_{k}^{*}\right)\right] \\
&-a_{k}^{2} w_{k}^{2}\left(2\left(d_{k}^{I}+\gamma_{k}\right)-\sigma_{3 k}^{2}-a_{k}\left(\epsilon_{k}+\gamma_{k}\right)\right)-a_{k}^{4} z_{k}^{2}\left(2 d_{k}^{R}-\sigma_{4 k}^{2}-a_{k} \gamma_{k}\right) \\
&\left.+2 \sum_{j=1}^{2} \beta_{k j} u_{k}\left(v_{k}-a_{k} u_{k}\right) \int_{r=0}^{\infty} f_{j}(r) v_{j}(t-r) d r+\sum_{j=1}^{2} \beta_{k j} S_{k}^{*}\left(1+a_{k}\right) v_{j}^{2}\right\} \\
&=L_{0} V+2 \sum_{k=1}^{2} \sum_{j=1}^{2} \beta_{k j} u_{k}\left(v_{k}-a_{k} u_{k}\right) \int_{r=0}^{\infty} f_{j}(r) v_{j}(t-r) d r,
\end{aligned}
$$

where

$$
\begin{aligned}
& L_{0} V \\
& =\sum_{k=1}^{2}\left\{-u_{k}^{2}\left[b_{k}\left(2 d_{k}^{S}-\sigma_{1 k}^{2}\right)-\sum_{j=1}^{2} a_{k} \beta_{k j} S_{k}^{*}\right]-v_{k}^{2}\left[2 b_{k} \sum_{j=1}^{2} \beta_{k j} S_{k}^{*}-\left(1+b_{k}\right) \sigma_{2 k}^{2}-a_{k}\left(\epsilon_{k}+\sum_{j=1}^{2} \beta_{k j} S_{k}^{*}\right)\right]\right. \\
& \left.\quad-a_{k}^{2} w_{k}^{2}\left(2\left(d_{k}^{I}+\gamma_{k}\right)-\sigma_{3 k}^{2}-a_{k}\left(\epsilon_{k}+\gamma_{k}\right)\right)-a_{k}^{4} z_{k}^{2}\left(2 d_{k}^{R}-\sigma_{4 k}^{2}-a_{k} \gamma_{k}\right)\right\} .
\end{aligned}
$$

From (4.9) it follows that there exists $a_{k}>0$ such that

$$
a_{k}<\min \left\{\frac{b_{k}\left(2 d_{k}^{S}-\sigma_{1 k}^{2}\right)}{\sum_{j=1}^{2} \beta_{k j} S_{k}^{*}}, \frac{2 b_{k} \sum_{j=1}^{2} \beta_{k j} S_{k}^{*}-\left(1+b_{k}\right) \sigma_{2 k}^{2}}{\epsilon_{k}+\sum_{j=1}^{2} \beta_{k j} S_{k}^{*}}, \frac{2\left(d_{k}^{I}+\gamma_{k}\right)-\sigma_{3 k}^{2}}{\epsilon_{k}+\gamma_{k}}, \frac{2 d_{k}^{R}-\sigma_{4 k}^{2}}{\gamma_{k}}\right\} .
$$

Therefore, there exists $\mathcal{C}>0$ such that $L V_{0} \leqslant-\mathcal{C}\left|\left(u_{k}, v_{k}, w_{k}, z_{k}\right)\right|^{2}$. 
Let us suppose that $P\left\{\left|v_{k}(t)\right|<\delta_{k}\right\}=1$. Then

$$
2 \sum_{j=1}^{2} \beta_{k j} u_{k}\left(v_{k}-a_{k} u_{k}\right) \int_{r=0}^{\infty} f_{j}(r) v_{j}(t-r) d r \leqslant \sum_{j=1}^{2} \beta_{k j} \delta_{k}\left(u_{k}^{2}\left(1+2 a_{k}\right)+v_{k}^{2}\right)
$$

Therefore

$$
\begin{aligned}
L V \leqslant & \sum_{k=1}^{2}\left\{-u_{k}^{2}\left[b_{k}\left(2 d_{k}^{S}-\sigma_{1 k}^{2}\right)-\sum_{j=1}^{2} a_{k} \beta_{k j} S_{k}^{*}-\sum_{j=1}^{2} \beta_{k j} \delta_{k}\left(1+2 a_{k}\right)\right]\right. \\
& -v_{k}^{2}\left[\left(1+b_{k}\right)\left(2 \sum_{j=1}^{2} a_{k} \beta_{k j} S_{k}^{*} h_{j}-\sigma_{2 k}^{2}\right)-a_{k}\left(\epsilon_{k}+\sum_{j=1}^{2} \beta_{k j} S_{k}^{*}\right)-\sum_{j=1}^{2} \beta_{k j} \delta_{k}\right] \\
& \left.-a_{k}^{2} w_{k}^{2}\left(2\left(d_{k}^{I}+\gamma_{k}\right)-\sigma_{3 k}^{2}-a_{k}\left(\epsilon_{k}+\gamma_{k}\right)\right)-a_{k}^{4} z_{k}^{2}\left(2 d_{k}^{R}-\sigma_{4 k}^{2}-a_{k} \gamma_{k}\right)\right\} .
\end{aligned}
$$

Hence for sufficiently small $\delta_{k}>0, L V(x, t)$ is negative definite in a sufficiently small neighborhood of $x=0$ for $t \geqslant 0$. According to Lemma 4.2, we therefore conclude that the zero solution of (4.2) is stochastically asymptotically stable in the large. The proof is complete.

\section{Acknowledgment}

The first author was partially supported by the NSF of China no. 10926106.

\section{References}

[1] M. Y. Li, Z. Shuai, and C. Wang, "Global stability of multi-group epidemic models with distributed delays," Journal of Mathematical Analysis and Applications, vol. 361, no. 1, pp. 38-47, 2010.

[2] H. Guo, M. Y. Li, and Z. Shuai, "A graph-theoretic approach to the method of global Lyapunov functions," Proceedings of the American Mathematical Society, vol. 136, no. 8, pp. 2793-2802, 2008.

[3] E. Beretta, V. Kolmanovskii, and L. Shaikhet, "Stability of epidemic model with time delays influenced by stochastic perturbations," Mathematics and Computers in Simulation, vol. 45, no. 3-4, pp. 269-277, 1998.

[4] N. Dalal, D. Greenhalgh, and X. Mao, "A stochastic model for internal HIV dynamics," Journal of Mathematical Analysis and Applications, vol. 341, no. 2, pp. 1084-1101, 2008.

[5] D. Greenhalgh, "Some results for an SEIR epidemic model with density dependence in the death rate," IMA Journal of Mathematics Applied in Medicine and Biology, vol. 9, no. 2, pp. 67-106, 1992.

[6] A. Korobeinikov, "Global properties of SIR and SEIR epidemic models with multiple parallel infectious stages," Bulletin of Mathematical Biology, vol. 71, no. 1, pp. 75-83, 2009.

[7] A. Korobeinikov and P. K. Maini, "A Lyapunov function and global properties for SIR and SEIR epidemiological models with nonlinear incidence," Mathematical Biosciences and Engineering, vol. 1, no. 1, pp. 57-60, 2004.

[8] L. S. Liebovitch and I. B. Schwartz, "Migration induced epidemics: dynamics of flux-based multipatch models," Physics Letters A, vol. 332, no. 3-4, pp. 256-267, 2004.

[9] W. O. Kermack and A. G. Mckendrick, "Contribution to the mathematical theory of epidemics," Proceedings of the Royal Society of London A, vol. 115, pp. 700-721, 1927.

[10] E. Tornatore, S. M. Buccellato, and P. Vetro, "Stability of a stochastic SIR system," Physica A, vol. 354, no. 1-4, pp. 111-126, 2005. 
[11] J. Yu, D. Jiang, and N. Shi, "Global stability of two-group SIR model with random perturbation," Journal of Mathematical Analysis and Applications, vol. 360, no. 1, pp. 235-244, 2009.

[12] L. Shaikhet, "Stability of predator-prey model with aftereffect by stochastic perturbation," Stability and Control: Theory and Applications, vol. 1, no. 1, pp. 3-13, 1998.

[13] M. Carletti, "On the stability properties of a stochastic model for phage-bacteria interaction in open marine environment," Mathematical Biosciences, vol. 175, no. 2, pp. 117-131, 2002.

[14] M. Bandyopadhyay and J. Chattopadhyay, "Ratio-dependent predator-prey model: effect of environmental fluctuation and stability," Nonlinearity, vol. 18, no. 2, pp. 913-936, 2005.

[15] R. R. Sarkar and S. Banerjee, "Cancer self remission and tumor stability—a stochastic approach," Mathematical Biosciences, vol. 196, no. 1, pp. 65-81, 2005.

[16] N. Bradul and L. Shaikhet, "Stability of the positive point of equilibrium of Nicholson's blowflies equation with stochastic perturbations: numerical analysis," Discrete Dynamics in Nature and Society, vol. 2007, Article ID 92959, 25 pages, 2007.

[17] M. Bandyopadhyay, T. Saha, and R. Pal, "Deterministic and stochastic analysis of a delayed allelopathic phytoplankton model within fluctuating environment," Nonlinear Analysis, vol. 2, no. 3, pp. 958-970, 2008.

[18] B. Paternoster and L. Shaikhet, "Stability of equilibrium points of fractional difference equations with stochastic perturbations," Advances in Difference Equations, vol. 2008, Article ID 718408, 21 pages, 2008.

[19] L. Shaikhet, "Stability of a positive point of equilibrium of one nonlinear system with aftereffect and stochastic perturbations," Dynamic Systems and Applications, vol. 17, no. 1, pp. 235-253, 2008.

[20] B. Mukhopadhyay and R. Bhattacharyya, "A nonlinear mathematical model of virus-tumor-immune system interaction: deterministic and stochastic analysis," Stochastic Analysis and Applications, vol. 27, no. 2, pp. 409-429, 2009.

[21] X. Mao, Stochastic Differential Equations and Applications, Horwood, Chichester, UK, 1997. 


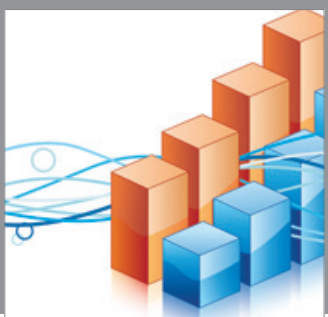

Advances in

Operations Research

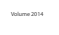

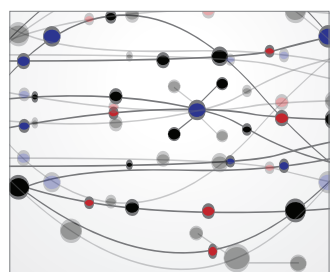

\section{The Scientific} World Journal
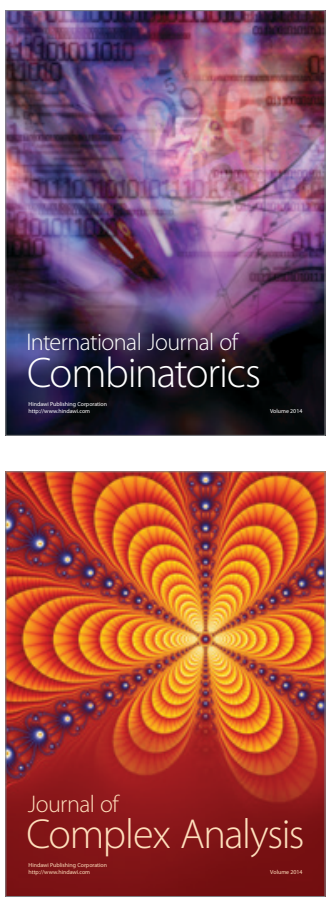

International Journal of

Mathematics and

Mathematical

Sciences
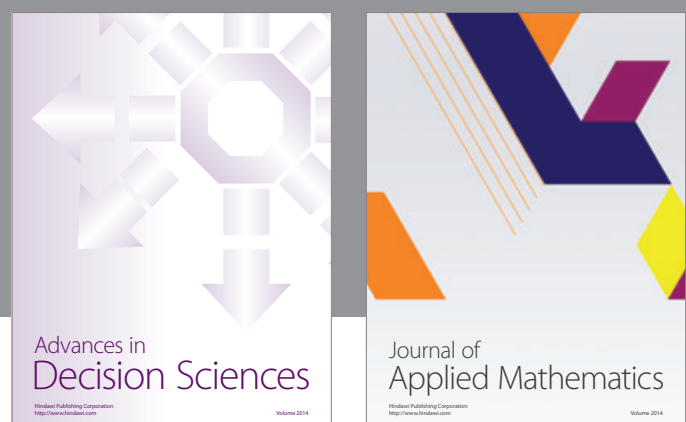

Journal of

Applied Mathematics
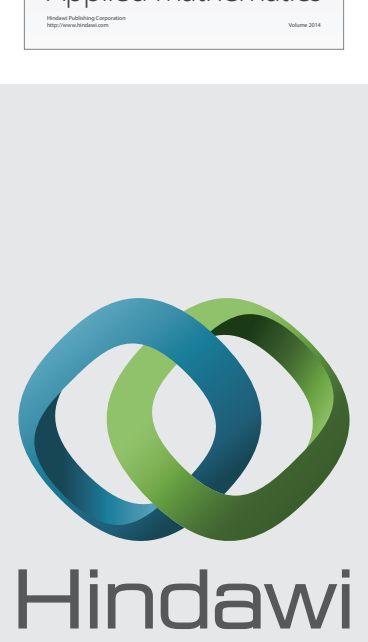

Submit your manuscripts at http://www.hindawi.com
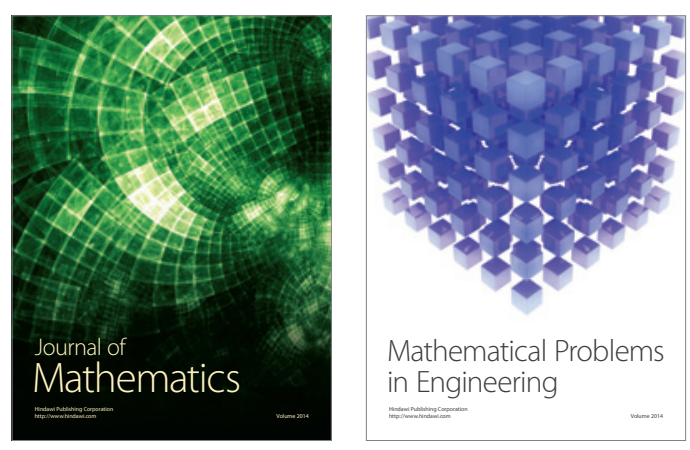

Mathematical Problems in Engineering
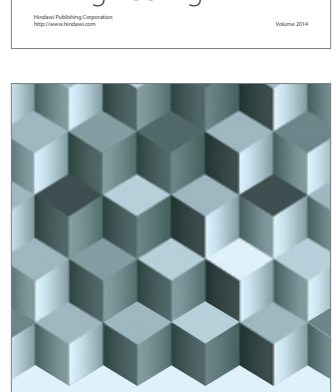

Journal of

Function Spaces
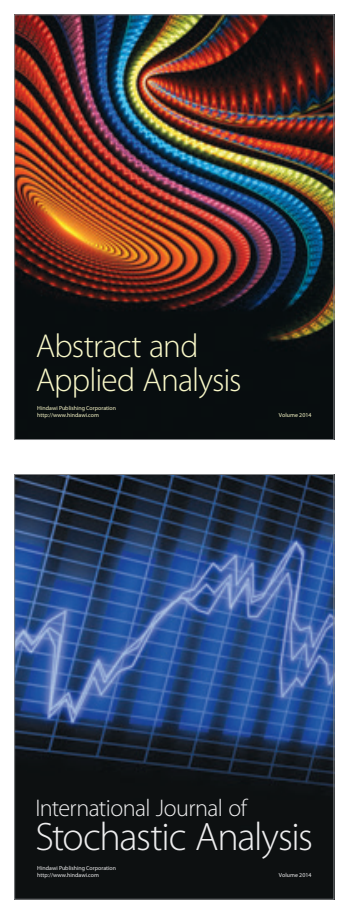

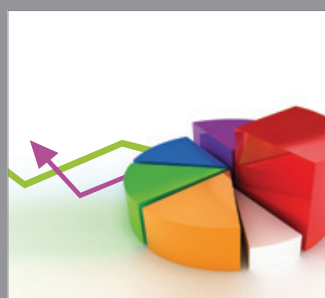

ournal of

Probability and Statistics

Promensencen
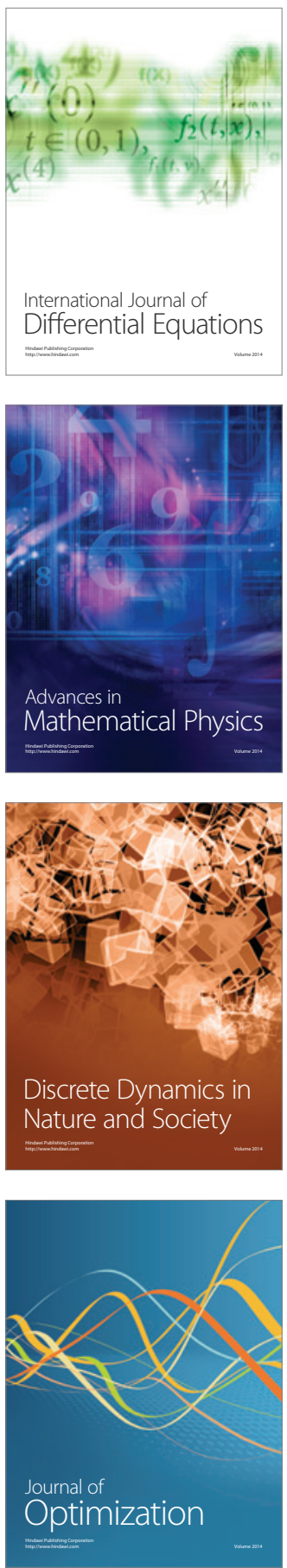